\title{
Perceived Barriers to Adherence to Tuberculosis Infection Control Measures among Health Care Workers in the Dominican Republic
}

\author{
Helena J. Chapman MD MPH PhD, Bienvenido A. Veras-Estévez MD MPH, Jamie L. Pomeranz MS PhD, \\ Eddy N. Pérez-Then MD MPH MSPH PhD, Belkys Marcelino MD ME-FETP, Michael Lauzardo MD MS
}

\begin{abstract}
INTRODUCTION Health care workers have an increased risk of infection due to occupational Mycobacterium tuberculosis exposure, including multidrug-resistant strains. Health care workers' risk of developing tuberculosis is greater than that of the general population, whether in low-, intermediate- or high-incidence countries. Adherence to infection control measures (administrative controls, environmental controls, and personal respiratory protection) is essential to reduce risk of disease transmission between suspected tuberculosis patients and health care workers, but for different reasons, both objective and subjective, adherence is low. Identifying the causes of low adherence is a prerequisite to effective programming to reduce risk.
\end{abstract}

OBJECTIVE Identify perceived barriers to adherence to tuberculosis infection control measures among health care workers in the Dominican Republic.

METHODS During August 2014, a qualitative study was conducted in two tertiary-level hospitals in different regions of the Dominican Republic. A semi-structured interview guide of nine questions was developed, based on the scientific literature and with consensus of clinical experts. Nine semi-structured interviews were conducted with a purposive sample of seven physicians (five men, two women) and two baccalaureate nurses (both women) working in the emergency medicine, internal medicine or nursing departments of those institu-

\section{INTRODUCTION}

Health care workers $(\mathrm{HCW})$ are at increased risk of infection due to occupational exposure to Mycobacterium tuberculosis, including multidrug-resistant strains. HCWs may be exposed and subsequently develop tuberculosis (TB) infection or active disease.[1] Compared to the general population, HCWs' incidence rate ratios ranged from 1.4 to 5.4 across low $(<50 / 100,000$ population), intermediate (50-99/100,000 population) and high (>100/100,000 population) TB incidence regions.[2] Compliance with infection control measures, recommended by $\mathrm{WHO}[3]$ and Centers for Disease Control and Prevention (CDC),[4] is essential to reduce risk of $M$. tuberculosis transmission between suspected TB patients and HCWs.

Published international guidelines for reducing nosocomial $M$. tuberculosis transmission have focused on a hierarchical strategy involving administrative controls (e.g., TB infection control plans; TB risk assessment; HCW training), environmental controls (e.g., ventilation to remove contaminated air) and personal respiratory protection (e.g., protective masks for HCWs and patients).[3,4] In 1999, WHO disseminated recommendations for TB infection control in low-resource health institutions,[3] and in 2005, CDC issued guidelines for preventing $M$. tuberculosis transmission in health care settings.[4] However, the elaborate infrastructure required to build, finance, manage and sustain such infection control strategies has limited their universal application in low-income countries.[5] Although these guidelines are ideal for health care tions. Question topics included clinical experience of $M$. tuberculosis infection and disease; knowledge of disease transmission and preventive practices; clinical management strategies; and perceptions of effectiveness of directly observed treatment, short-course, and disease coping strategies.

RESULTS Perceived barriers were described as: 1) sense of invincibility of health care workers; 2 ) personal beliefs of health care workers related to direct patient communication; 3) low provider-topatient ratios in hospitals; 4) absence of tuberculosis isolation units for patients within hospitals; and 5) limited availability of protective masks for health care workers.

CONCLUSIONS Our results highlight that perceived barriers at the individual or institutional level may hinder how health care workers understand and comply with preventive strategies to reduce risk of tuberculosis transmission. Addressing these barriers by strengthening infection control program infrastructure and implementing educational interventions within institutions may reduce risk of nosocomial tuberculosis transmission to health care workers.

KEYWORDS Health care providers, infection control, infectious disease transmission, health care associated infection, nosocomial infection, Mycobacterium tuberculosis occupational exposure, occupational health, qualitative research, tuberculosis, Dominican Republic

settings, they fail to consider perceived barriers of HCWs, on the frontlines of TB care.

Academic coursework and supervised clinical rotations provide $\mathrm{HCWs}$, such as physicians and nurses, with knowledge and skills in TB pathology, diagnosis and management. They should, therefore, understand their occupational risk of $M$. tuberculosis exposure and the importance of adhering to recommended $M$. tuberculosis infection control measures. However, in limited-resource settings, ineffective infection control strategies, coupled with HCWs' erroneous understanding or perceptions of their occupational risk of M. tuberculosis exposure, can challenge HCWs' ability to adhere to recommended $M$. tuberculosis infection control measures in their clinical practice.[6]

The Dominican Republic (DR) is a middle-income country in the Caribbean with intermediate TB incidence (60/100,000 in 2015).[7] Directly observed treatment, short course (DOTS), was introduced in 1999[8] and reached $94 \%$ of the population by 2012 ,[9] with a demonstrable reduction in TB incidence, from 92 per 100,000 in 2002 to 60 per 100,000 in 2014.[9,10] However, high levels of multidrug resistance (3\% in new cases; $12 \%$ in previously treated cases in 2015) and incidence of TB-HIV coinfection (15/100,000 in 2015) have been reported.[7] This TB burden may influence occupational exposure for HCWs in contact with patients infected with susceptible or resistant $M$. tuberculosis strains, especially patients with immunocompromising conditions such as AIDS. According to one 
study, 111 HCWs (31 baccalaureate nurses, 20 janitors, 13 physicians, 8 certificate-level nurses, 7 laboratory personnel, 6 maintenance crew, 4 administrative personnel, 1 dentist and 21 other/unknown) at 49 provincial and regional hospitals developed active TB disease between 2005 and 2012.[11]

The DR National Tuberculosis Program has actively revised and widely disseminated their guidelines, based on WHO and CDC recommendations, to enhance TB prevention and control across DR communities and health institutions.[9,12] Currently, no established national active surveillance programs or regular medical evaluations for $\mathrm{HCW}$ s report the number of $\mathrm{HCW}$ sho become exposed and develop latent TB infection (LTBI) or active TB. However, in 2001, the Dominican Social Security System approved Law No. 87-01 for protection of all DR citizens in the workplace.[13] In 2004, the Dominican Social Security Institute developed the Occupational Health Risk Insurance plan, providing financial restitution for injury or disease caused by occupational accidents or exposures.[13] In 2008, the DR Ministry of Public Administration adopted Law No. 41-08, providing for paid medical leave to employees during the designated recovery period.[14] Thus, although physicians and nurses rely on this federal and institutional support in the workplace and are clinically trained in disease management and prevention, they should be prepared to apply their knowledge about $M$. tuberculosis infection control measures to clinical practice.

With reported evidence of HCWs who developed active TB disease at tertiary-level DR hospitals,[11] it is essential to understand how HCWs perceive their risk of occupational $M$. tuberculosis exposure and report use of preventive measures in their clinical practice. By examining the "knowledge-action" gap among HCWs, described as their inability to apply their clinical knowledge to practice,[15] their perceived barriers can be identified and subsequently addressed by health authorities in order to reduce occupational risk of nosocomial $M$. tuberculosis transmission.

Although there have been advancements in TB diagnostics, treatment and prevention, little qualitative research has been done. Such research could add value to collaborative methods for global TB control.[16] The study purpose was to identify, using a qualitative approach, perceived barriers to adherence to TB infection control measures among HCWs in the DR.
Table 1: Characteristics of study hospitals, Dominican Republic, 2014

\begin{tabular}{|c|c|c|}
\hline Characteristic & Hospital A & Hospital B \\
\hline \multicolumn{3}{|l|}{ Context } \\
\hline City & Santo Domingo & Santiago de los Caballeros \\
\hline Population (2010) & $2,374,370$ & 963,422 \\
\hline \multicolumn{3}{|l|}{ General } \\
\hline Category & National & Regional \\
\hline Year opened & 1946 & 1978 \\
\hline Annual admissions & 11,513 & 24,822 \\
\hline Annual emergency visits & 168,485 & 105,337 \\
\hline Annual consultations & 117,110 & 175,759 \\
\hline Beds* $^{*}$ & 214 & 425 \\
\hline \multicolumn{3}{|l|}{ Medical training programs } \\
\hline Medical specialty programs & 6 & 10 \\
\hline Medical subspecialty programs & 2 & 13 \\
\hline \multicolumn{3}{|l|}{ Tuberculosis } \\
\hline Cases with respiratory symptoms & 1,062 & 524 \\
\hline \multicolumn{3}{|l|}{ TB cases diagnosed } \\
\hline Patients & 139 & 15 \\
\hline Health care workers & 4 & 1 \\
\hline \multicolumn{3}{|l|}{ MDR-TB cases diagnosed } \\
\hline Patients & 0 & 0 \\
\hline Health care workers & 0 & 0 \\
\hline \multicolumn{3}{|l|}{ Presence of TB isolation area } \\
\hline Internal medicine ward & No & No \\
\hline Intensive care unit & No & No \\
\hline
\end{tabular}

Source: Dominican Republic Ministry of Health National Department of Statistics, National Tuberculosis Program, and Hospital B

*Due to hospital renovations by the Dominican Republic Ministry of Health, number of hospital beds varied by month in 2014. Thus, we summed the monthly numbers and divided by 12 to calculate the monthly average.

MDR: multidrug-resistant TB: tuberculosis

\section{METHODS}

Setting and sample During August 2014, a qualitative study was conducted in two tertiary-level hospitals in different regions of the DR (Hospital A in Santo Domingo and Hospital B in Santiago de los Caballeros). Characteristics of study hospitals are displayed in Table 1. A purposive sample was selected of nine HCWs, seven of whom were attending or resident physicians in internal or emergency medicine, and two nurses, all involved in TB prevention, control or management.
Table 2: Participant characteristics

\begin{tabular}{r|c|l|l|l|l|}
\hline No. & Hospital & \multicolumn{1}{|c|}{ Profession } & Sex & \multicolumn{1}{|c|}{ Department } & \multicolumn{1}{c|}{ Title } \\
\hline 1 & A & Physician & Male & Emergency medicine & Attending physician (chief) \\
\hline 2 & A & Physician & Male & Emergency medicine & Attending physician \\
\hline 3 & A & Physician & Male & Internal medicine & Attending physician \\
\hline 4 & B & Physician & Male & Internal medicine & Fourth-year resident (chief) \\
\hline 5 & A & Physician & Male & Internal medicine & Fourth-year resident \\
\hline 6 & A & Physician & Female & Internal medicine & Attending physician (chief) \\
\hline 7 & A & Physician & Female & Internal medicine & Attending physician \\
\hline 8 & A & Baccalaureate nurse & Female & Nursing & Nursing coordinator \\
\hline 9 & B & Baccalaureate nurse & Female & Nursing & Nursing director \\
\hline
\end{tabular}




\section{Original Research}

and disease management in the hospital (available online at www .medicc.org/mediccreview/chapman.html). The guide was based on the scientific literature, with consensus of clinical experts. We defined perceived barriers according to the health behavior model, as elements of a specific health action that negatively influence adherence to recommended actions.[18] Question topics included clinical experience related to $M$. tuberculosis infection and disease; b) knowledge of disease transmission and preventive practices; c) clinical management strategies; and d) perceptions of effectiveness of DOTS and disease coping strategies.

For demographic purposes, participants described their educational and clinical responsibilities within the institution. Questions were written in English and translated into Spanish, and then verified by a bilingual Dominican physician. Each interview was conducted in Spanish by the first author, a general physician trained in the DR, and digitally recorded at the main desk of the closest clinical ward or in a private office. Interview duration varied depending on length of participant responses. Field notes were made by hand after completion of each interview. Data collection was finalized when no new emerging themes were observed, thus reaching data saturation.[19]

Analysis Data were transcribed and de-identified by the first author and verified by one co-author. QSR International's NVivo 10 qualitative data analysis software (QSR International Inc., Burlington, MA, USA) was used to manage transcripts and facilitate coding. Based on knowledge and experience in TB, both researchers developed initial categories prior to the interviews. They coded interview transcripts separately and then met to discuss all coded nodes and discrepancies to enhance interobserver reliability. Using thematic analysis, they analyzed these coded notes and developed emerging themes with illustrative quotations.[20,21] Card sorting and peer debriefing were used to ensure reliability and validity.[20,22] A descriptive and illustrative model was developed to represent emerging themes about perceived barriers.

Ethics This study was approved by the Institutional Review Boards of the University of Florida (USA) and O\&M Medical School (DR). The protocol was reviewed and authorized by the DR National Tuberculosis Program. Finally, the protocol was approved by Hospital A's Department of Academics and Hospital B's Institutional Review Board. Written informed consent was obtained from all participants.

\section{RESULTS}

Interview duration ranged from 9 to 25 minutes (average 15). The following perceived barriers emerged: 1) HCWs' sense of invincibility; 2) HCWs' personal beliefs about direct patient communication; 3) low providerto-patient ratios; 4) absence of TB isolation units for patients in hospitals; and 5) limited availability of protective masks for HCWs. Figure 1 presents the illustrative model of the emerging themes.

HCWs' sense of invincibility Most participants conveyed only minor concern for nosocomial TB transmission, alluding to prior $M$. tuberculosis exposure from $B C G$ vaccine, clinical exposure in their short- or longterm employment at the hospital, or both.
One nurse mentioned that all HCWs most likely have LTBI: I imagine that all [health care workers] who have worked in clinical wards, I believe we all have [TB] sensitivity.

One male physician echoed this sentiment that all physicians will probably have a positive result to a tuberculin skin test, suggesting that previous $M$. tuberculosis exposure has resulted in LTBI:

Normally, we [physicians] are positive for tuberculosis [infection]. In the majority of health centers, physicians who work in these centers will have a positive result to the tuberculin skin test. We do not have prophylaxis for this. Well, we take care of ourselves enough, some of us.

In fact, two physicians used humor to express their belief that little could be done if the entire country has been infected with $M$. tuberculosis. One male physician stated:

Here in the Dominican Republic, we represent 10 million people who have a positive result of the tuberculin skin test. How can we act when the source [M. tuberculosis] is next to us?

One female physician mentioned:

The majority of the population [laughs] of this country, you can classify as latent tuberculosis. What are we going to do with this group? We are not going to do anything because the majority of the population has it [latent tuberculosis], where I even include myself.

Three participants mentioned that they did not develop TB disease, even though they had clinical responsibilities caring for TB patients. One nurse mentioned:

I worked for five years on the women's clinical ward with patients who were diagnosed with TB. I did not even develop the flu [laughs].

However, two participants commented that they have observed that HCWs had developed TB disease due to confirmed occupational exposure and successfully completed prescribed treatment regimens. One nurse stated:

Here, we have also had nurses who have been released from the center and were confirmed cured [of tuberculosis].

Figure 1: Conceptual model of barriers to HCWs' compliance with guidelines to reduce occupational MTB exposure
Low provider-to-patient ratio Absence of patient isolation units Limited availability of protective masks

Institutional-level barriers

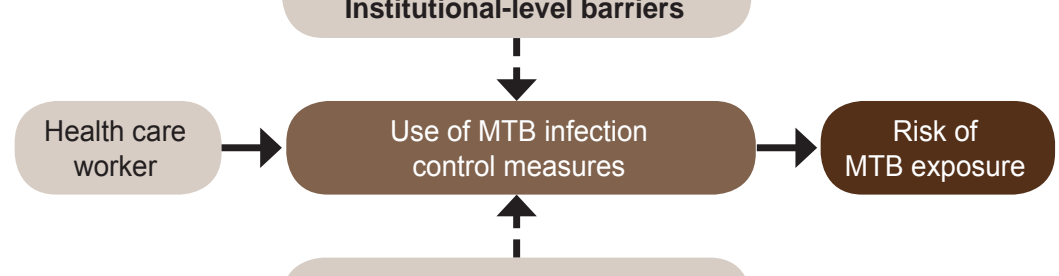

Individual-level barriers

Sense of invincibility

Personal beliefs related to direct patient communication
HCW: health care worker MTB: M. tuberculosis 
Participants mentioned that one primary preventive measure is placing protective masks on TB patients. One nurse commented that, aside from the BCG vaccination used as one preventive method within the institution, these protective masks are fundamental measures:

[We must place] masks on patients in order to protect the health care worker.

However, one male physician remained optimistic and added that $\mathrm{HCW}$ are knowledgeable and conscious of the TB transmission cycle and do not want to continue transmission to family members at home.

HCWs' personal beliefs about direct patient communication Physicians and nurses emphasized that, as HCWs, they genuinely aim to provide the best attention and quality of clinical care services to patients. They expressed, however, that their personal beliefs about direct patient communication influence how they choose to protect themselves from possible occupational exposure to $M$. tuberculosis. In one example, the protective respiratory mask was seen as a physical barrier interfering with effective provider-patient dialogue. One male physician observed that, when caring for patients in the Emergency Department, empathy and building patient rapport take priority over wearing personal protective equipment such as a mask:

It is more cultural than anything. It looks ugly when you are in the Emergency Department. I put on a mask as if I am going to become infected [by a patient]. It looks ugly, so we do not do this. We do, however, keep our distance from patients and avoid speaking closely face-to-face.

In a second example, spiritual faith was considered to play a protective role for HCWs during provider-patient interactions during medical appointments and ward rounds, whether considered as an organized religion or a source of hope and peace, this invisible form of defense from $M$. tuberculosis transmission was one last protective measure. One female physician described her clinical experiences with direct care of TB patients, crediting her spiritual faith for that fact that she had not developed TB:

When we are examining patients, we do not use any type of preventive measures for TB transmission in patients who have the bacillus... counting on the divine presence to help us [laughs] with the prevention, unfortunately.

Low provider-to-patient ratios Physicians and nurses were knowledgeable about the high burden of TB in the DR and the clinical protocol for patients with respiratory symptoms seeking medical services. They noted that the typical flow of patients to be assessed for TB at medical clinics is high. In particular, one physician stated that her clinic receives new and followup cases for assessment and management of TB disease:

The day-to-day [flow] of persons who arrive as new cases... in our clinic, I see approximately 20 to 25 patients, just as [name of physician], that we function here in the department, with new patients ranging from one to five in each clinic... and then we see followup tuberculosis cases.

Another female physician expanded on this to illustrate increased admission to clinical wards of probable or confirmed TB cases, often occupying the hospital's entire bed capacity:

Right now, of eight patients admitted to the medical ward, four are probable tuberculosis, two are confirmed tuberculosis. The patient who just arrived is probable tuberculosis. Many times, the entire clinical ward of eight beds for men and eight beds for women is full of tuberculosis.

Participants mentioned that many patients who develop respiratory symptoms will self-medicate with over-the-counter antibiotics or home remedies, thus leaving their disease undetected and untreated and continuing to transmit $M$. tuberculosis. They stated that TB patients commonly present with severe clinical manifestations, which are associated with increased risk of severe consequences or death. One female physician emphasized that TB patients who remain undiagnosed in the community will succumb to the disease if they are not assessed and admitted to the hospital for aggressive TB management:

...but here, we generally treat tuberculosis that is advanced, I mean, the patient comes, and if you do not admit the patient [to the hospital], he or she will die.

Absence of TB isolation units for patients in hospitals Participants responded that their hospitals did not have appropriate isolation units to separate probable or confirmed TB patients from other patients of other diseases. They were aware that the absence of this environmental control compromised their personal protection and placed them at increased risk of $M$. tuberculosis exposure. One nurse described the current scenario in her hospital as less than ideal:

One thing is what should be done and another is what we can do, because what should be done is that there would be an isolation area [for the patient], but sometimes it is not like that...

One male physician expressed that HCWs, on the front line for M. tuberculosis exposure, lacked the necessary protective measures to reduce nosocomial TB transmission between patients and HCWs or even between patients:

We live in a country with high transmission of pulmonary TB, and in this health center, we do not have the appropriate protective resources or a specific isolation unit in order to control [tuberculosis] transmission.

In turn, another male physician further illustrated how the absence of a TB isolation unit in the hospital may place other patients at risk for nosocomial TB transmission:

We have a clinical ward where we admit patients with respiratory diseases... patients with TB, chronic obstructive pulmonary disease, lung cancer, etc. So, when I place a patient with pulmonary TB [in the room], I am putting the other patients at risk.

Limited availability of protective masks for HCWs Physicians and nurses recognized that health institutions had limited budgets to manage daily functions, including administrative, clinical and laboratory responsibilities. Participants mentioned that protective masks were inconsistently available for use by HCWs or patients. One female physician described the protective function of masks when they are available in the clinical environment:

[Protective masks] are ideal, but you know, the costs of masks should be provided by the government. Here, we do not have masks. We are not provided masks, unless you bring a cloth mask.

One nurse mentioned that she was quite aware of the limited protection conferred by surgical-type masks in her hospital:

The masks that we have are the surgical type, which do not protect us [as health care workers] but rather them [patients]. 
We place these masks on them [patients], and thus, we are 'half-way' protected [laughs].

Recognizing that TB is one of multiple diseases that HCWs encounter in their workplace, one nurse admitted that HCWs are unable to fully protect themselves from nosocomial $M$. tuberculosis transmission. She mentioned that her hospital had not prioritized the use of specialized masks as the best preventive approach to reduce possible $M$. tuberculosis transmission among HCWs and patients:

We should use the N95 mask, but all health personnel, physicians like nurses, use the simple [surgical] mask. We use real 'prevention, prevention' when we know that there is a tuberculosis patient. We take precautionary measures. But in the general sense, what should be done [in reality] is using the correct mask and having an isolation area for patients, which [in our case] we lack.

\section{DISCUSSION}

This is the first known study to explore HCWs' perceived barriers to adherence to TB infection control measures in DR hospitals. As a country with intermediate TB incidence, TB incidence rates have decreased over the last decade.[9,10] However, four common challenges in TB control have been reported by the DR National Tuberculosis Program: reaching marginalized populations living in high-burden provinces; assessing influence of domestic (rural-urban) and international migration (from Haiti); understanding social determinants of health associated with impoverished living conditions; and promoting community participation in "Estrategia Alto a la TB" initiatives that reduce TB-associated stigma and discrimination in vulnerable populations. [9] HCWs' occupational exposure, however, remains overlooked and continues to be driven by increased utilization of medical services by persons who are undiagnosed and untreated for susceptible or resistant M. tuberculosis strains.

In our study, participants reported individual-level barriers that they perceive as hindering their ability to adhere to recommended $M$. tuberculosis infection control measures. They expressed the opinion that exposure to $M$. tuberculosis is inevitable, whether because of prior BCG vaccination or their duration of employment in health care. This presents a three-fold challenge. First, although BCG vaccines have been shown to provide immunological protection against advanced TB disease in children, such protection is estimated to last $<10$ years, and there is no protective effect in adults.[23,24] Second, HCWs reported feeling reassured that they had not developed TB, but only the common cold or flu, even though employed in TB-endemic settings. Thus, they may erroneously perceive that their habitual occupational exposure to the pathogen has produced immunity against developing TB disease.[6] Third, HCWs described the essential concept of the "human touch" in direct patient communication, aiming to improve patient rapport by forgoing protective masks and relying on avoiding close contact with patients, a basic but less effective protective measure. Thus, HCWs may decide to relinquish personal protection in order to maximize perceived communication and compassionate care to patients while reducing associated stigma.[25] However, HCWs who fail to adhere to recommended M. tuberculosis infection control measures may not be upholding their ethical and moral responsibility to protect their own as well as their patients' health.
They also reported perceived institutional-level barriers that reflected limited availability of institutional funding for appropriate and effective protection against $M$. tuberculosis for HCWs. Failure to promptly diagnose and implement appropriate infection control measures for suspected TB patients is a fundamental factor raising risk of nosocomial M. tuberculosis transmission. [4] Since nosocomial M. tuberculosis transmission in HCWs has been documented by the DR Ministry of Health,[11] dual challenges will need to be addressed. First, although successful DOTS rollout reached an estimated $94 \%$ national coverage by 2012,[9] interrelated personal, health service, social and structural factors continue to complicate patient adherence to long-term $M$. tuberculosis pharmaceutical treatment.[26] Thus, HCWs may be exposed to M. tuberculosis by erroneously assuming that TB patients seeking health care services are compliant with treatment. Second, neither pre-employment nor annual screening of HCWs for LTBI with the tuberculin skin test or interferon-gamma release assay is routinely conducted in the DR. Without such regular testing, this high-risk group may not receive LTBI diagnosis and recommended preventive therapy to reduce the estimated $5-10 \%$ lifetime risk of reactivation TB.[27] Without systematic assessment of nosocomial $M$. tuberculosis transmission in HCWs, health institutions will be unable to effectively prioritize HCWs' physical health.

A knowledge-action gap, defined as the observed inconsistent application of clinical knowledge in direct clinical practice,[15,28] can increase risk of occupational exposure to pathogens or negatively affect delivery of health care services.[15] Participants reported daily occupational exposure to $M$. tuberculosis, but they did not always take steps to protect themselves, evidence of a knowledge-action gap. Airborne protection measures (appropriate masks and isolation units) are essential in such institutions, but a series of factors that expand said gap have been documented in both low- and high-income countries: time-constrained or short-staffed health settings, HCWs' limited knowledge or erroneous perceptions, lack of access to clinical research, political ideologies that conflict with scientific evidence, and lack of financial resources.[15,29]

In conducting the study, we documented that no national active surveillance system existed for the timely reporting of HCWs who develop TB infection or disease or who do not adhere to $M$. tuberculosis infection control measures. The only national evidence identifying risk of this occupational health threat was the study cited above, reporting that $111 \mathrm{HCW}$ developed TB between 2005 and 2012.[11] Passive surveillance is not sufficient to promptly detect TB cases or identify inadequate infection control practices. The DR needs to invest in an electronic database that connects each local health institution with the National Tuberculosis Program, so that data from active surveillance systems can be reviewed immediately to identify local and national TB incidence and monitor HCWs' adherence to infection control measures.

This study identified five perceived barriers among HCWs that limit their ability to comply with strategies to reduce risk of TB transmission, providing a starting point for in-depth study of these barriers, aimed at building a theoretical framework. Future studies can use theoretical sampling and multiple data collection methods[19] to examine HCWs' perceptions of specific intrinsic and extrinsic processes that influence their clinical decisionmaking regarding $M$. tuberculosis infection control measures. In 
turn, this framework can be validated among HCWs employed in other hospitals and environments (e.g., rural areas; low- or intermediate-incidence countries; primary- or secondary-level hospitals). Thus, stakeholders and other leaders of health institutions can, in collaboration with HCWs, tailor interventions and develop practical recommendations to promote HCWs' adherence to TB infection control measures.

In addition, by illuminating underlying cultural processes, the framework can serve as a baseline for future quantitative or mixed-methods research designs to test hypotheses and identify causal associations between selected variables. One such example could be to assess the effectiveness of implementation of the recommended hierarchical infection control strategy in institutional $M$. tuberculosis infection control programs.

This study had some limitations, among them a small sample size. The fact that we reached data saturation, however, suggests that the sample size was sufficient.[19] Second, the first author conducted all interviews as an external member of both hospitals, which may have inhibited participation. However, with hospital administration buy-in, in the form of academic and research support, the first author gained rapport and trust with participants, thus reducing any potential bias. Third, this study intended to explore overall clinical knowledge, perspectives and infection control practices among HCWs about TB infection and disease related to transmission, diagnosis, management and prevention. Thus, in-depth exploration and review, rather than generalizability, were essential to examine the cultural processes related to delivery of health care services.
Finally, although two researchers reviewed the data, it should be recognized that there may be additional ways to interpret the data and more variables to explore in future study designs to validate our results.[30]

\section{CONCLUSIONS}

Using a qualitative approach, this study identified perceived barriers related to adherence to TB infection control measures among HCWs in the DR. Our findings suggest that ineffective TB infection control strategies coupled with erroneous understanding or perceptions of TB risk complicate TB management in limitedresource settings. Perceived barriers at the individual or institutional level may hinder how HCWs understand the actual risk of TB transmission and are able to comply with preventive strategies to reduce risk of $M$. tuberculosis exposure. Addressing such barriers by strengthening infection control program infrastructure and implementing educational interventions within institutions may reduce HCWs' risk of nosocomial TB transmission.

\section{ACKNOWLEDGMENTS}

The authors thank the study participants for sharing their experiences and perspectives on TB control in the DR. We appreciate research support from the DR National Tuberculosis Program, O\&M Medical School and selected hospitals. We acknowledge the academic mentorship of Dr. Mary Ellen Young and peer debriefing provided by students of the Qualitative Data Analysis course (PHC6937) and University of Florida Qualitative Research Colloquium throughout the data analysis process. We also appreciate logistical support from Dr. Nelson Bencosme, Dr. Josefina Bruno, Dr. Kreilin López, and Saulo Rodríguez. - 1/

\section{REFERENCES}

1. Menzies D, Joshi R, Pai M. Risk of tuberculosis infection and disease associated with work in health care settings. Int J Tuberc Lung Dis. 2007 Jun;11(6):593-605.

2. Baussano I, Nunn P, Williams B, Pivetta E, Bugiani M, Scano F. Tuberculosis among health care workers. Emerg Infect Dis. 2011 Mar;17(3):488-94

3. World Health Organization. Guidelines for the prevention of tuberculosis in health care facilities in resource-limited settings. Geneva: World Health Organization; 1999. $51 \mathrm{p}$.

4. Jensen PA, Lambert LA, lademarco MF, Ridzon $\mathrm{R}$; CDC. Guidelines for preventing the transmission of Mycobacterium tuberculosis in healthcare settings, 2005. MMWR Recomm Rep. 2005 Dec 30;54(RR-17):1-141.

5. Pai M, Kalantri S, Aggarwal AN, Menzies D, Blumberg HM. Nosocomial tuberculosis in India. Emerg Infect Dis. 2006 Sep;12(9):1311-8.

6. von Delft A, Dramowski A, Khosa C, Kotze $\mathrm{K}$, Lederer $\mathrm{P}$, Mosidi $\mathrm{T}$, et al. Why healthcare workers are sick of TB. Int J Infect Dis. 2015 Mar;32:147-51.

7. World Health Organization. Tuberculosis country profile: Dominican Republic, 2015 [Internet]. Geneva: World Health Organization; 2016 [cited 2016 Dec 1]. Available from: https://extranet.who .int/sree/Reports?op=Replet\&name $=\% 2 \mathrm{FWHO}$ HQ Reports\%2FG2\%2FPROD\%2FEXT\%2FT $\bar{B}$ CountryProfile\&ISO2=DO\&LAN=EN\&outtype $=$ html

8. Pérez-Then E, Acosta I, Marcelino B, Espinal M. Tuberculosis in the Dominican Republic: addressing the barriers to sustain the achievements. Bull World Health Organ. 2007 May;85(5):384-5.
9. Ministry of Health (DO). Reglamento técnico para la prevención y el control de la tuberculosis [Internet]. Santo Domingo: Ministry of Health (DO); 2014 [cited 2016 Dec 1]. 38 p. Report No.: MSP/ VGC-RT02-14. 58 p. Available from: http://www .sespas.gov.do/oai/Documentos/Reglamentos/ REGL_TecPrevContrlolTuberculosis_20140819 .pdf. Spanish.

10. World Health Organization. Global Health Observatory data repository. Tuberculosis: Incidence data by country [Internet]. Geneva: World Health Organization. 2015 [cited 2016 Dec 1]. Available from: http://apps.who.int/gho/data/node. main.1320?lang=en

11. Genao M, Rodríguez A. Prevalencia de enfermedad tuberculosa en trabajadores de salud a nivel nacional, República Dominicana, 2005-2012. In: Programa National de Tuberculosis. Investigación operativa en tuberculosis, 2011-2013: dossier de investigaciones. Santo Domingo: Ministry of Health (DO); 2013. p. 195-205. Spanish

12. Ministry of Health (DO). Normas Nacionales para la Prevención y Control de la Tuberculosis. Serie de Normas Nacionales No. 16. 5th ed [Internet]. Santo Domingo: Ministry of Health (DO); 2010 [cited 2016 Jul 21]. 64 p. Available from: http://saludysociedadcivil.org.do/index.php/ documentos/47/Nacional/142/Normas Naciona les_para_el_Control_de_la_Tuberculosis_2010 .pdf. Spanish.

13. Occupational Health and Safety Administration (DO). Carta compromiso al ciudadano [Internet]. Santo Domingo: Occupational Health and Safety Administration (DO); 2015 [cited 2016 Jul 21]. 29 p. Available from: http://www
arlss.gov.do/PaginasDinamicas/Informacio nes/Boletines/p01/presentacion_No_01.pdf. Spanish.

14. Ministry of Public Administration (DO). Ley No. 41-08 sobre Función Pública: Ley No. 4108 de Función Pública y crea la Secretaría de Estado de Administración Pública [Internet]. Santo Domingo: Ministry of Public Administration (DO); 2008. 38 p. Available from: http:// observatorioserviciospublicos.gob.do/base legal/Ley-41-08-Funcion-publica.pdf. Spanish.

15. Haines A, Kuruvilla S, Borchert M. Bridging the implementation gap between knowledge and action for health. Bull World Health Organ. 2004 Oct:82(10):724-31.

16. Engel N, Pai M. Tuberculosis diagnostics: why we need more qualitative research. J Epidemiol Glob Health. 2013 Sep;3(3):119-21.

17. Centers for Disease Control and Prevention. Tuberculosis behavioral and social science research forum: planting the seeds for future research. Proceedings of the Tuberculosis Behavioral and Social Science Research Forum [Internet]; 2003 Dec 10-11; Georgia, United States. Atlanta: U.S. Department of Health and Human Services, Centers for Disease Control and Prevention; 2005 Mar 1 [cited 2016 Aug 5]. 129 p. Available from: http://www.cdc.gov/tb/topic/ research/images_pdf/forum_proceedings.pdf

18. Skinner CS, Tiro J, Champion VL. The health belief model. In: Glanz K, Rimer BK, Viswanath $K$, editors. Health behavior: theory, research, and practice. 5th ed. San Francisco (US): JosseyBass; 2015. p. 75-94. 
19. Charmaz K. Constructing grounded theory. 2nd ed. Los Angeles: Sage Publications, Inc.; 2014 Apr 9.416 p.

20. Ryan GW, Bernard HR. Techniques to identify themes. Field Methods. 2003 Feb 1;15(1):85109.

21. Sandelowski M, Barroso J. Classifying the findings in qualitative studies. Qual Health Res. 2003 Sep;13(7):905-23.

22. Lincoln YS, Guba EG. Naturalistic inquiry. Newbury Park (US): Sage Publications; 1985 Apr. 416 p.

23. Abubakar I, Pimpin L, Ariti C, Beynon R, Mangtani P, Sterne JA, et al. Systematic review and meta-analysis of the current evidence on the duration of protection by bacillus Calmette-Guérin vaccination against tuberculosis. Health Technol Assess. 2013 Sep;17(37):1-372.

24. Andersen P, Woodworth JS. Tuberculosis vaccines-rethinking the current paradigm. Trends Immunol. 2014 Aug;35(8):387-95.

25. Brouwer M, Coelho E, Mosse Cd, Brondi L, Winterton L, van Leth F. Healthcare workers challenges in the implementation of tuberculosis infection prevention and control measures in Mozambique. PLoS One. 2014 Dec;9(12):e114364.

26. Munro SA, Lewin SA, Smith $\mathrm{HJ}$, Engel ME, Fretheim A, Volmink J. Patient adherence to tuberculosis treatment: a systematic review of qualitative research. PLoS Med. 2007 Ju 24:4(7):e238

27. Zumla A, Raviglione $M$, Hafner R, von Reyn CF. Tuberculosis. N Engl J Med. 2013 Feb;368(8):745-55.

28. Graham ID, Logan J, Harrison MB, Straus SE Tetroe J, Caswell W, et al. Lost in knowledge translation: time for a map? J Contin Educ Health Prof. 2006 Winter;26(1):13-24.
29. Farmer PE. Shattuck lecture. Chronic infectious disease and the future of health care delivery. $\mathrm{N}$ Engl J Med. 2013 Dec;369(25):2424-36.

30. Miles MB, Huberman AM, Saldaña J. Qualitative data analysis: a methods sourcebook. 3rd ed. Thousand Oaks (US): Sage Publications, Inc.; 2013 Apr 18. 408 p.

\section{THE AUTHORS}

Helena J. Chapman (Corresponding author: hchapman@ufl.edu), physician specializing in public health, with a master's degree in public health (epidemiology) and doctorate in public health (One Health). Research scientist, Division of Infectious Diseases and Global Medicine, University of Florida College of Medicine (UFCM), Gainesville, Florida, USA.

Bienvenido A. Veras-Estévez, physician specializing in epidemiology, with a master's degree in public health. Coordinator, Department of Research, Universidad Católica Tecnológica del Cibao Faculty of Health Sciences, La Vega, Dominican Republic. Coordinator, Department of Epidemiology and Statistics, Hospital Regional Universitario José María Cabral y Báez, Santiago, Dominican Republic.

Jamie L. Pomeranz, certified rehabilitation counselor and life care planner, with master's degrees in exercise and sports science and rehabilitation counseling, and a doctorate in rehabilitation science. Associate professor and researcher, Department of Occupational Therapy, University of Florida College of Public Health and Health Professionals, Gainesville, Florida, USA.

Eddy N. Pérez-Then, physician specializing in public health, with master's degrees in public health and global health and doctorate in global health. Director, professor and researcher, O\&M Medical School, Santo Domingo, Dominican Republic.

Belkys Marcelino, physician specializing in epidemiology, with a master's degree in field epidemiology training programs. Director, National Tuberculosis Program, Santo Domingo, Dominican Republic.

Michael Lauzardo, internist and pulmonologist with a master's degree in epidemiology. Chief and associate professor, Division of Infectious Diseases and Global Medicine, UFCM, Gainesville, Florida, USA.

Submitted: October 19, 2016

Approved for publication: January 5, 2017 Disclosures: None

\section{NURSING 2017}

\section{7th Congress of the Cuban Nursing Society}

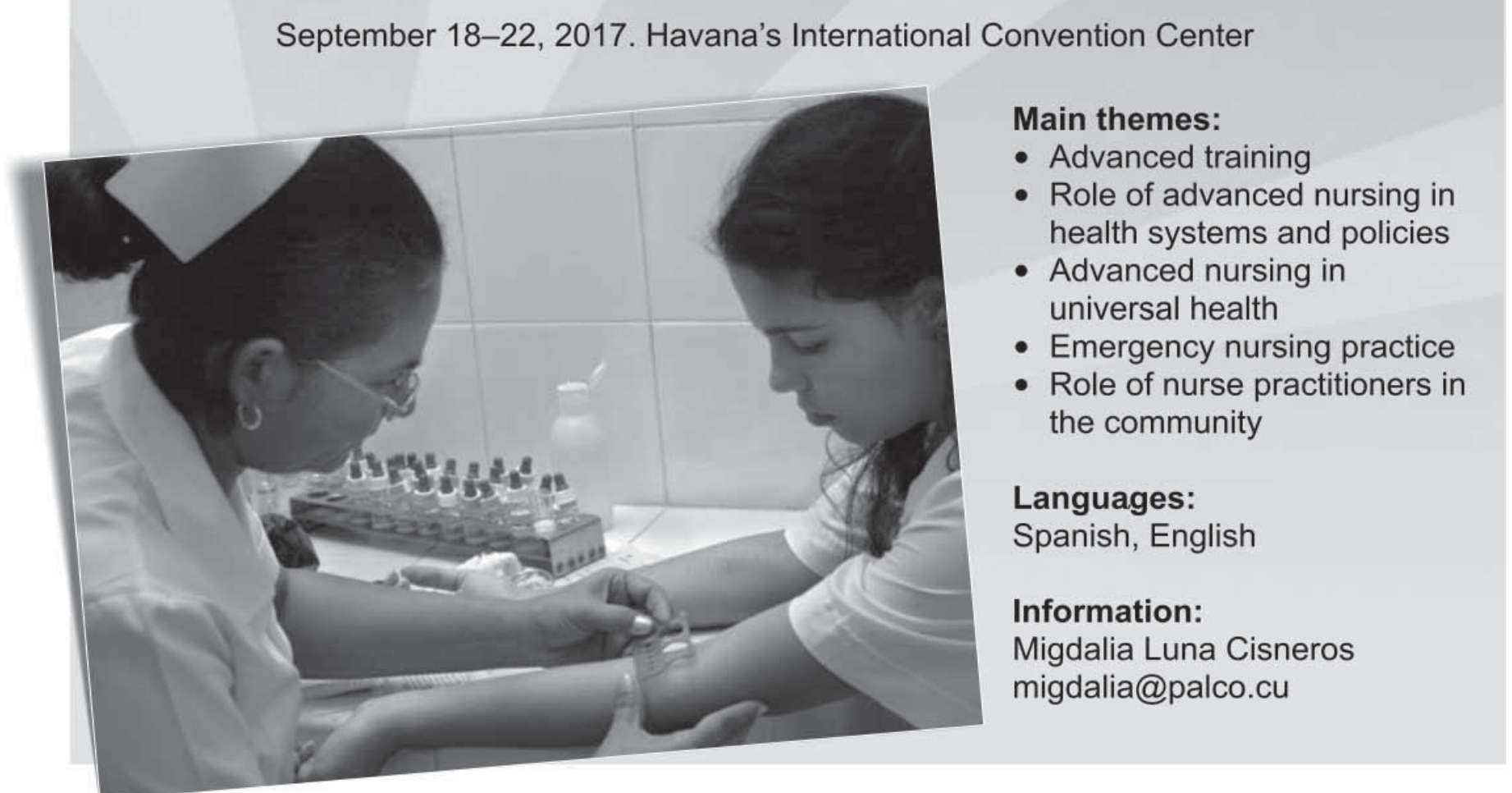

